\title{
Construction of Non-Gaussian Self-Similar Random Fields with Hierarchical Structure
}

\author{
P. M. Bleher \\ Keldysh Institute of Applied Mathematics, Miusskaya Square, 4, Moscow A-47, USSR
}

\begin{abstract}
In the present work we construct non-Gaussian self-similar random fields with hierarchical structure. The construction is based on non-Gaussian solutions of the main nonlinear equation of the hierarchical models theory. The existence of such solutions was proved originally by Sinai and the author and later by another method by Collet and Eckmann. Next we establish the uniqueness of a Gibbs state for the constructed self-similar field. Finally for a class of hierarchical models we prove the convergence of renormalization transformations of a random field at the critical point to the self-similar field.
\end{abstract}

\section{Definitions}

Let $r \in \mathbb{Z}, r \geqq 2$, and $\xi_{0}>\xi_{1}>\xi_{2}>\ldots$ be a decreasing sequence of partitions of a countable set $V$ satisfying the following conditions:

(i) $\xi_{0}$ is the partition of $V$ into separate points,

(ii) any element of the partition $\xi_{n}$ consists of $r$ elements of the partition $\xi_{n-1}$, $n=1,2, \ldots$,

(iii) for any two points $i, j \in V$ a number $n$ exists such that $i, j$ belong to the same element of the partition $\xi_{n}$.

Such a sequence of partitions is called a hierarchical structure in the set $V$ (see [1]). Let us denote $n(i, j)$ the least number $n$ such that $i, j$ belong to the same element of the partition $\xi_{n}$. The quantity

$$
d(i, j)=\left\{\begin{array}{lll}
0 & \text { if } & i=j \\
r^{n(i, j)} & \text { if } & i \neq j
\end{array}\right.
$$

defines a metrics in a set $V$ with hierarchical structure.

A map $V \rightarrow V^{\prime}$ is called an isomorphism of hierarchical structures if it preserves the structure of the partitions. One can see easily that for a given $r$ any two hierarchical structures are isomorphic. We shall consider two realizations of the 
hierarchical structure:

(i) $V=\mathbb{Z}, \xi_{n}=\left\{V_{n k}, k \in \mathbb{Z}\right\}$, $V_{n k}=\left\{i \mid-r^{n-1}+k r^{n} \leqq i<-r^{n-1}+(k+1) r^{n}\right\}$.

(ii) $V=\left\{\alpha=0 \cdot \alpha_{1} \alpha_{2} \ldots \mid 0 \leqq \alpha_{i}<r, \exists N: \alpha_{i}=0\right.$ if $\left.i \geqq N\right\}$, $\xi_{n}=\left\{V_{n \beta}, \beta \in V\right\}$, $V_{n \beta}=\left\{\alpha=0 \cdot \alpha_{1} \alpha_{2} \ldots \mid \alpha_{n+i}=\beta_{i}, i=1,2, \ldots\right\}$.

[ $V$ is the set of finite $r$-mal fractions and $V_{n \beta}$ are the sets of such fractions with fixed tails; it is noteworthy that in this realization $d(i, j)$ is the usual $p$-adic metrics if $r=p$ is a prime.]

As a rule we shall use the first realization. A configuration $\sigma$ in a volume $V^{0} \mathrm{CV}$ is an arbitrary real-valued function $\sigma=\sigma(i)$ on $V^{0}$. The set of all configurations in a volume $V^{0} C V$ is denoted by $\sum\left(V^{0}\right)$. The Hamiltonian of the hierarchical model $(\mathrm{HM})$ in a volume $V_{n k}$ is given by the formula

where the potential

$$
H_{n k}(\sigma)=\sum_{\substack{(i, j): i \neq j \\ i, j \in V_{n k}}} U(i, j) \sigma(i) \sigma(j),
$$

$$
U(i, j)=-d(i, j)^{-a} .
$$

The quantity $a>1$ is a parameter of the HM. Sometimes we shall use $c=r^{2-a}$ instead of $a$ as a parameter of the HM. A boundary condition for $V_{n k}$ is a configuration (real-valued function) $\sigma^{\prime}=\sigma^{\prime}(i)$ on $V \backslash V_{n k}$ such that for $i \in V_{n k}$ the series

$$
h\left(\sigma^{\prime}\right)=\sum_{j \in V \backslash V_{n k}} U(i, j) \sigma^{\prime}(j)
$$

is absolutely converging. Let us note that $h\left(\sigma^{\prime}\right)$ does not depend on $i$. The HM Hamiltonian with a boundary condition $\sigma^{\prime}$ is defined as

$$
H_{n k}\left(\sigma \mid \sigma^{\prime}\right)=H_{n k}(\sigma)+\sum_{i \in V_{n k}} \sum_{j \in V \backslash V_{n k}} U(i, j) \sigma(i) \sigma^{\prime}(j) .
$$

Let $\Lambda$ be the space of probability distributions $v(d x)$ on $\mathbb{R}^{1}$ such that

$$
\int_{-\infty}^{\infty} \exp \left(A x^{2}\right) v(d x)<\infty \quad \forall A \in \mathbb{R}^{1} .
$$

For $v \in \Lambda$ we define the Gibbs distribution of the HM in a volume $V_{n k}$ with a boundary condition $\sigma^{\prime}$ as

$$
\begin{aligned}
& \mu_{n k}\left(d \sigma \mid \beta, v, \sigma^{\prime}\right)= \Xi_{n k}^{-1}\left(\beta, v, \sigma^{\prime}\right) \exp \left[-\beta H_{n k}\left(\sigma \mid \sigma^{\prime}\right)\right] \\
& \cdot \prod_{i \in V_{n k}} v(d \sigma(i)), \\
& \Xi_{n k}\left(\beta, v, \sigma^{\prime}\right)=\int \exp \left[-\beta H_{n k}\left(\sigma \mid \sigma^{\prime}\right)\right] \prod_{i \in V_{n k}} v(d \sigma(i)) .
\end{aligned}
$$

A limit distribution of the HM is defined as

$$
\lim _{n \rightarrow \infty} \mu_{n 0}\left(d \sigma \mid \beta, v, \sigma^{\prime}\right)=\mu(d \sigma \mid \beta, v)
$$


(if the limit exists) for a sequence of boundary conditions $\sigma^{\prime}$ [in general $\mu(d \sigma \mid \beta, v)$ depends on the sequence of $\sigma^{\prime}$ ]. The limit is understood as the weak convergence of the finite dimensional distributions. Let us denote $\Pi_{0}$ the set of finite permutations of $V$ which preserve the hierarchical structure. Any limit Gibbs distribution is invariant with respect to any permutation $\pi_{0} \in \Pi_{0}$.

Let $\mu(d \sigma)$ be a probability measure on $\sum\left(V_{n 0}\right)$ or $\sum(V)$. The joint distribution of the random variables

$$
\bar{\sigma}(k)=r^{-\frac{a m}{2}} \sum_{i \in V_{m k}} \sigma(i), V_{m k} \subset V_{n 0} \quad \text { (or } V_{m k} \subset V \text { respectively), }
$$

defines a probability measure $\mu_{m}(d \bar{\sigma})$ on $\sum\left(V_{n-m, k}\right)$ [or $\sum(V)$ respectively]. The measure $\mu_{m}(d \bar{\sigma})$ is called a renormalization transformation (RT) of the measure $\mu(d \sigma)$ (see [4]) and is denoted $\mu_{m}(d \sigma)=R_{r^{m}, n}^{(a)} \mu(d \sigma)$ [or $=R_{r^{m}}^{(a)} \mu(d \sigma)$ respectively]. A probability measure $\mu(d \sigma)$ on $\sum(V)$ is called self-similar (other names: scaling invariant, automodel) if $R_{r m}^{(a)} \mu(d \sigma)=\mu(d \sigma), m=1,2, \ldots$. It is noteworthy that $R_{r^{m}}^{(a)}=\left(R_{r}^{(a)}\right)^{m}$, so, for self-similarity, it is sufficient that $R_{r}^{(a)} \mu(d \sigma)=\mu(d \sigma)$ (for details of the definitions see [4]).

\section{Results}

Let us define a non-linear operator $v \rightarrow Q_{a, \beta}(v)$ in the space $\Lambda$ such that for any Borel set $B \subset \mathbb{R}^{1}$

$$
Q_{a, \beta}(v)(B)=L^{-1} \underset{\frac{x_{1}+\ldots+x_{r}}{r^{a / 2}} \in B}{\int \ldots \int} \exp \left[\beta A\left(x_{1}, \ldots, x_{r}\right)\right] \prod_{i=1}^{r} v\left(d x_{i}\right),
$$

where

$$
\begin{gathered}
A\left(x_{1}, \ldots, x_{r}\right)=r^{-a} \sum_{(i, j): i \neq j} x_{i} x_{j}, \\
L=\int_{-\infty}^{\infty} \ldots \int_{-\infty}^{\infty} \exp \left[\beta A\left(x_{1}, \ldots, x_{r}\right)\right] \prod_{i=1}^{r} v\left(d x_{i}\right) .
\end{gathered}
$$

Theorem 1. Let $\mu(d \sigma \mid \beta, v)$ be a limit Gibbs distribution of the HM, $v \in \Lambda$. Then $R_{r}^{(a)} \mu(d \sigma \mid \beta, v)=\mu\left(d \sigma \mid \beta, v^{\prime}\right)$ where $v^{\prime}=Q_{a, \beta}(v)$.

Remark. If $\mu(d \sigma \mid \beta, v)$ is a limit of the Gibbs distributions $\mu_{n 0}\left(d \sigma \mid \beta, v, \sigma_{n}^{\prime}\right)$ with respect to a sequence of boundary conditions $\sigma_{n}^{\prime} \in \sum\left(V \backslash V_{n 0}\right), n=n_{1}, n_{2}, \ldots$, then $R_{r}^{(a)} \mu(d \sigma \mid \beta, v)$ is a limit of the Gibbs distributions $\mu\left(d \sigma \mid \beta, v^{\prime}, \bar{\sigma}_{n}^{\prime}\right)$ with respect to the sequence of normalized mean boundary conditions

$$
\left\{\bar{\sigma}_{n}^{\prime}(i)=r^{-a / 2} \sum_{j \in V_{1,2}} \sigma_{n}^{\prime}(j), V_{1, i} \subset V \backslash V_{n 0}\right\} .
$$

In the papers $[2,3]$, for $a=2-1 / j+\varepsilon, 0<\varepsilon<\varepsilon_{j}, \varepsilon_{j}>0, j=2,3, \ldots$, non-Gaussian functions $p_{j, \varepsilon}^{*}(x) \in \mathscr{S}\left(\mathbb{R}^{1}\right), p_{j, \varepsilon}^{*}(x)>0, \int_{-\infty}^{\infty} p_{j, \varepsilon}^{*}(x) d x=1$, were constructed such that the measures $v_{j, \varepsilon}^{*}(d x)=p_{j, \varepsilon}^{*}(x) d x$ are fixed points of the operator $Q_{a, 1}$ :

$$
Q_{a, 1}\left(v_{j, \varepsilon}^{*}\right)=v_{j, \varepsilon}^{*} .
$$


One can see easily that then $v_{j, \varepsilon, \beta}^{*}(d x)=p_{j, \varepsilon}^{*}(x \sqrt{\beta}) d x \sqrt{\beta}$ are fixed points of the operator $Q_{a, \beta}$ :

$$
Q_{a, \beta}\left(v_{j, \varepsilon, \beta}^{*}\right)=v_{j, \varepsilon, \beta}^{*} .
$$

The functions $p_{j, \varepsilon}^{*}(x)$ can be written in the form

$$
p_{j, \varepsilon}^{*}(x)=L^{-1} \exp \left[-a_{0} x^{2}-\varphi_{j, \varepsilon}^{*}(x)\right]
$$

where $a_{0}=(r-1) /\left(r^{a}-r\right)$. The functions $\varphi_{j, \varepsilon}^{*}(x) \in C^{\infty}\left(\mathbb{R}^{1}\right)$ satisfy the conditions:

$$
\left\|\varphi_{j, \varepsilon}^{*}(x)-\varepsilon H_{j}(x)\right\|_{C^{3}([-|\ln \varepsilon|,|\ln \varepsilon|])}<C \varepsilon^{3 / 2},
$$

where $H_{j}(x)$ is a polynomial of degree $2 j$ and, for some constant $C_{j}>0$ and $|x|>\frac{1}{2}|\ln \varepsilon|$

$$
\left|\frac{\frac{d^{k}}{d x^{k}} \varphi_{j, \varepsilon}^{*}(x)}{C_{j} \varepsilon \alpha(\alpha-1) \ldots(\alpha-k+1)|x|^{\alpha-k}}-1\right|<C \varepsilon^{1 / 2}
$$

where $\alpha=(2-a)^{-1}, k=0,1,2,3\left[C_{j}\right.$ coincides with the senior coefficient of the polynomial $\left.H_{j}(x)\right]$. The condition $(2.5)$ gives a description of the behaviour of $\varphi_{j, \varepsilon}^{*}(x)$ for finite $x$ while (2.6) describes the asymptotics of $\varphi_{j, \varepsilon}^{*}(x)$ when $x \rightarrow \infty$.

Theorem 2. Let $a=2-1 / j+\varepsilon, 0<\varepsilon<\varepsilon_{j}$. The Gibbs distributions $\mu_{n 0}\left(d \sigma \mid \beta, v_{j, \varepsilon, \beta}^{*}, \sigma_{n}^{\prime}\right)$ with zero boundary condition $\sigma_{n}^{\prime}=\left\{\sigma_{n}^{\prime}(i) \equiv 0\right\}$ converge to a self-similar limit Gibbs distribution $\mu\left(d \sigma \mid \beta, v_{j, \varepsilon, \beta}^{*}\right)$.

The next theorem is the most difficult one in the present paper.

Theorem 3. Let $a=2-1 / j+\varepsilon, 0<\varepsilon<\varepsilon_{j}$. For any sequence of boundary conditions $\sigma_{n}^{\prime}=\left\{\sigma_{n}^{\prime}(i)\right\}$ such that $h\left(\sigma_{n}^{\prime}\right)_{n \rightarrow \infty} 0$ the Gibbs distributions $\mu_{n 0}\left(d \sigma \mid \beta, v_{j, \varepsilon, \beta}^{*}, \sigma_{n}^{\prime}\right)$ converge to a self-similar limit Gibbs distribution which does not depend on the sequence $\sigma_{n}^{\prime}$.

The condition $h\left(\sigma_{n}^{\prime}\right)=\sum_{j \in V \backslash V_{n 0}} d^{-a}(i, j) \sigma_{n}^{\prime}(j)_{n \rightarrow \infty} 0$ is extremely important. If $h\left(\sigma_{n}^{\prime}\right)_{n \rightarrow \infty} h \neq 0$ then the limit still exists but it is a limit Gibbs distributions of the HM in the presence of the external magnetic field $h$. The existence of the limit in this case can be proved by the same method as we use in the proof of the Theorem 3. By a limit Gibbs distribution in the presence of a magnetic field $h$ we mean one whose conditional distributions are defined by the Hamiltonians $H_{n k}\left(\sigma \mid \sigma^{\prime}\right)+h \sum_{i \in V_{n k}} \sigma(i)$. In other words this is a Gibbsian distribution in the sense of the Dobrushin-Lanford-Ruelle (DLR) equations (see $[5,6]$ ) with the HM Hamiltonian with the external field $h$.

Theorem 3 allows us to obtain the uniqueness theorem of the DLR equations for the self-similar field constructed in Theorem 2. Now we formulate such a theorem.

Theorem 4. $\mu\left(d \sigma \mid \beta, v_{j, \varepsilon, \beta}^{*}\right)$ is the unique Gibbs distribution in the sense of the DLR equations. 
In the papers $[2,3]$ it was proved also that for $a=3 / 2+\varepsilon, 0<\varepsilon<\varepsilon_{2}$, the measure $v_{2, \varepsilon, \beta}^{*}(d x)$ which is a fixed point of the transformation $Q_{a, \beta}$ arises as a limit of iterations $v^{(n+1)}=Q_{a, \beta}\left(v^{(n)}\right), v^{(0)}=v$, at the critical point $\beta=\beta_{c}=\beta_{c}(v)$ of the HM for an open (in some natural topology) set of measures $v(d x) \in \Lambda$. The fixed points $v_{j, \varepsilon, \beta}^{*}(d x)$ with $j>2$ appear in the theory of multicritical phenomena. Namely, $v_{j, \varepsilon, \beta}^{*}(d x), j>2$ appear as a rule, as a limit of iterations of $Q_{a, \beta}$ at the critical point $\beta=\beta_{c}$, when applied to a $(j-2)$-parametrical family of measures $v_{\alpha}(d x)$, $\alpha=\left(\alpha_{1}, \ldots, \alpha_{j-2}\right)$ for a certain value $\alpha=\alpha^{0}$. The corresponding theorem is proved in the same way as for $j=2$ (see $[2,3]$; a good discussion of multicritical phenomena in the HM is contained in [7]). There are two classes of measures $v(d x)$ for which the existence of the critical point can be established: discrete measures on a lattice (see [2] where asymptotically hierarchical models are dealt with) and measures absolutely continuous with respect to Lebesque measure on $\mathbb{R}^{1}$ (see [3]). Here we consider the second case. In such a case a $(j-2)$-parametrical family of measures $v_{\alpha}(d x)=p_{\alpha}(x) d x, p_{\alpha}(x) \in \mathscr{S}\left(\mathbb{R}^{1}\right)$,

$$
0<p_{\alpha}(x)<C_{1} \exp \left(-a_{0} x^{2}-C_{2} \varepsilon x^{2 j}\right)
$$

is constructed such that there exists the (unique) value $\alpha=\alpha^{c}$ for which at the critical point $\beta=\beta_{c}$ the iterations $Q_{a, \beta_{c}}^{n}\left(v_{\alpha^{c}}\right)=p^{(n)}(x) d x$ converge to the measure $v_{j, \varepsilon, \beta_{c}}^{*}(d x)$, this statement being true for small perturbations of the family $v_{\alpha}(d x)$. For the sequence of the functions $p^{(n)}(x)$ the convergence takes place in the sense that

$$
\sup _{|x|<\sqrt{n}}\left|p^{(n)}(x)-p_{j, \varepsilon, \beta_{c}}^{*}\right|<\lambda^{n}, \quad 0<\lambda<1
$$

and constants $C_{1}, C_{2}>0$ exist such that

$$
0<p^{(n)}(x)<C_{1} \exp \left(-a_{0} x^{2}-C_{2} \varepsilon x^{2 j}\right) .
$$

Theorem 5. Let $a=2-1 / j+\varepsilon, 0<\varepsilon<\varepsilon_{j}$, and a function $p^{(0)}(x)$ be given such that for the functions $p^{(n)}(x)$ defined by the equation $v^{(n)}=Q_{\alpha, \beta_{c}}^{n}\left(v^{(0)}\right), v^{(n)}(d x)=p^{(n)}(x) d x$, $n=0,1,2, \ldots$, the conditions (2.7), (2.8) are fulfilled. Then for zero boundary conditions a limit Gibbs distribution $\mu\left(d \sigma \mid \beta_{c}, v^{(0)}\right)$ exists. Moreover

$$
\lim _{n \rightarrow \infty} R_{r^{n}}^{(a)} \mu\left(d \sigma \mid \beta_{c}, v^{(0)}\right)=\mu\left(d x \mid \beta_{c^{\prime}}, v_{j, \varepsilon, \beta_{c}}^{*}\right) .
$$

\section{Proofs}

For simplicity we shall consider the case $r=2$. The extension to the general case is straightforward.

The Main Formula. Let $\mu_{n}(d \sigma \mid \beta, v, 0)$ be the Gibbs distribution in a volume $V_{n 0}$ with zero boundary conditions and $\gamma_{n}\left(d \xi_{n}\right)$ be the probability distribution of the random variable

$$
\xi_{n}=2^{-\frac{n a}{2}} \sum_{i \in V_{n 0}} \sigma(i)
$$


with respect to $\mu_{n}(d \sigma \mid \beta, v, 0)$. Let $N \geqq n$ and $\mu_{N}\left(\mathrm{~d} \sigma \mid \beta, v, \sigma^{\prime}\right)$ be the Gibbs distribution in the volume $V_{N 0}$ with boundary condition $\sigma^{\prime}$. Let finally $\mu_{n N}\left(d \sigma \mid \beta, v, \sigma^{\prime}\right)$ be the joint distribution of the variables $\sigma(i), i \in V_{n 0}$, with respect to $\mu_{N}\left(d \sigma \mid \beta, v, \sigma^{\prime}\right)$. Then

$$
\mu_{n N}\left(d \sigma \mid \beta, v, \sigma^{\prime}\right)=L^{-1} f_{n N}\left(\xi_{n}\right) \mu_{n}(d \sigma \mid \beta, v, 0),
$$

where $\xi_{n}$ is defined by (3.1) and

$$
f_{n N}(x)=T_{n+1} \cdots T_{N} \exp \left(\beta 2^{n a / 2} h\left(\sigma^{\prime}\right) x\right),
$$

where $h\left(\sigma^{\prime}\right)$ was introduced in (1.3) and

$$
\begin{aligned}
T_{m}: f(x) \rightarrow \frac{\sqrt{c}}{2} \int \exp \left(\frac{\beta c}{4} x y\right) f\left(\frac{\sqrt{c}}{2}(x+y)\right) \gamma_{m}(d y), \\
c=2^{2-a} .
\end{aligned}
$$

$L$ is a normalizing factor in (3.2).

Proof of the Main Formula. Let $V_{m 0}^{\prime}=V_{m+1,0} \backslash V_{m 0}$. To compute $\mu_{n N}\left(d \sigma \mid \beta, v, \sigma^{\prime}\right)$ one has to integrate $\mu_{N}\left(d \sigma \mid \beta, v, \sigma^{\prime}\right)$ in the variables $\sigma(i), i \in V_{N 0} \backslash V_{n 0}=V_{n}^{\prime} \cup \ldots \cup V_{N-1}^{\prime}$. This will be done in two steps. At first we fix the values of the variables

$$
\eta_{m}=2^{-\frac{m a}{2}} \sum_{i \in V_{m}^{\prime}} \sigma(i)=2^{a / 2} \xi_{m+1}-\xi_{m},
$$

$m=n, \ldots, N-1$ and integrate in all $\sigma(i)$ under fixed $\eta_{m}, m=n, \ldots, N-1$. As a result we come to the probability distribution

$$
\begin{gathered}
L^{-1} \mu_{n}(d \sigma \mid \beta, v, 0) \prod_{m=n}^{N-1}\left[\exp \left(\frac{\beta c}{4} \xi_{m} \eta_{m}\right) \gamma_{m}\left(d \eta_{m}\right)\right] \\
\cdot \exp \left[\beta 2^{N a / 2} h\left(\sigma^{\prime}\right) \xi_{N}\right] .
\end{gathered}
$$

Next we integrate in the variables $\eta_{N-1}, \ldots, \eta_{n}$ and it gives the main formula (3.2). The proof complete.

As a matter of fact the formula (3.2) was used implicitly in the paper [8]. It shows that the random variables $\xi_{m}, m=N, \ldots, n$, form a (in general nonstationary) Markov chain. For further use we note that for $N=n$ the formula (3.2) is written as

$$
\mu_{N}\left(d \sigma \mid \beta, v, \sigma^{\prime}\right)=L^{-1} \exp \left[\beta 2^{N a / 2} h\left(\sigma^{\prime}\right) \xi_{N}\right] \mu_{N}(d \sigma \mid \beta, v, 0) .
$$

Proof of Theorem 1. At first we prove that

$$
R_{2, N}^{(a)} \mu_{N}(d \sigma \mid \beta, v, 0)=\mu_{N-1}\left(d \sigma \mid \beta, v^{\prime}, 0\right)
$$

By (1.1), (1.2) we have:

$$
H_{N 0}(\sigma)=H_{N-1,0}(\bar{\sigma})-\sum_{j: V_{1 j} \subset V_{N 0}} 2^{-a} \prod_{i \in V_{1 j}} \sigma(i)
$$

where

$$
\bar{\sigma}=\left\{\bar{\sigma}(j)=2^{-a / 2} \sum_{i \in V_{1},} \sigma(i), V_{1 j} \subset V_{N 0}\right\}
$$


Hence

and

$$
\begin{aligned}
& \mu_{N}(d \sigma \mid \beta, v, 0)=\Xi_{N}^{-1}(\beta, v) \exp \left[-\beta H_{N 0}(\sigma)\right] \prod_{i \in V_{N 0}} v(d \sigma(i)) \\
& \quad=\Xi_{N}^{-1}(\beta, v) \exp \left[-\beta H_{N-1,0}(\bar{\sigma})+\beta 2^{a} \sum_{j: V_{1} \subset V_{N O}} \sigma(i)\right] \prod_{i \in V_{N O}} v(d \sigma(i))
\end{aligned}
$$

$$
\begin{aligned}
R_{2, N}^{(a)} \mu_{N}(d \sigma \mid \beta, v, 0)= & \Xi_{N}^{-1}(\beta, v) \exp \left[-\beta H_{N-1,0}(\bar{\sigma})\right] \\
& \cdot \prod_{j: V_{1 j} \subset V_{N 0}}\left[L^{-1} Q_{a, \beta}(v)(d \bar{\sigma}(j))\right] \\
= & \Xi_{N-1}^{-1}\left(\beta, v^{\prime}\right) \exp \left[-\beta H_{N-1,0}(\bar{\sigma})\right] \\
& \cdot \prod_{j: V_{1 j} \subset V_{N 0}} v^{\prime}(d \bar{\sigma}(j))=\mu_{N-1}\left(d \bar{\sigma} \mid \beta, v^{\prime}, 0\right),
\end{aligned}
$$

which was stated.

Using (3.6) we can extend Eq. (3.7) to arbitrary boundary conditions:

$$
R_{2, N}^{(a)} \mu_{N}\left(d \sigma \mid \beta, v, \sigma^{\prime}\right)=\mu_{N-1}\left(d \sigma \mid \beta, v^{\prime}, \bar{\sigma}^{\prime}\right)
$$

where $\bar{\sigma}^{\prime}=\left\{\bar{\sigma}^{\prime}(j)=2^{-a / 2} \sum_{i \in V_{1 j}} \sigma^{\prime}(i), V_{1 j} \not V_{N 0}\right\}$. If the left hand side of this equation has a limit for a sequence $\left\{\sigma_{N}^{\prime}\right\}$ of boundary conditions, then the right hand side converges for the corresponding sequence $\left\{\bar{\sigma}_{N}^{\prime}\right\}$ and the limits coincide. Theorem 1 is proved.

Proof of Theorem 2. It is sufficient to consider $\beta=1$. The general case is reduced to this one by the change $\sigma(i) \rightarrow \sqrt{\beta} \sigma(i)$. Therefore we do not indicate the dependence on $\beta$. Our aim is to prove the convergence of finite-dimensional distributions $\mu_{n N}\left(d \sigma \mid v_{j, \varepsilon}^{*}, \sigma^{\prime}\right)$ to a limit when $N \rightarrow \infty$. We use the main formula (3.2). The distribution $\mu_{n}\left(d \sigma \mid v_{j, \varepsilon}^{*}, 0\right)$ does not depend on $N$ so the theorem will be proved if we establish the convergence of the functions $f_{n N}(x) / f_{n N}(0)$ to a limit. To prove the convergence we use the formula (3.3).

If $\gamma_{n}=\gamma_{n}\left(d \xi_{n}\right)$ is the distribution of the random variable $\xi_{n}$ [see (3.1)] with respect to $\mu_{n}\left(d \sigma \mid v_{j, \varepsilon}^{*}, 0\right)$, then by $(1.1),(1.2)$

Moreover

$$
\gamma_{n+1}=Q_{a}\left(\gamma_{n}\right) \text {. }
$$

$$
\gamma_{0}=v_{j, \varepsilon}^{*}
$$

so by virtue of (2.2)

$$
\gamma_{n}=Q_{a}^{n}\left(v_{j, \varepsilon}^{*}\right)=v_{j, \varepsilon}^{*}, n=0,1,2, \ldots,
$$

all the operators $T_{n+1}, \ldots, T_{N}$ coincide with

$$
\begin{aligned}
T: f(x) & \rightarrow \frac{\sqrt{c}}{2} \int \exp \left(\frac{c}{4} x y\right) f\left(\frac{\sqrt{c}}{2}(x+y)\right) v_{j, \varepsilon}^{*}(d y) \\
& =\int \exp \left[\frac{c}{4} x\left(\frac{2}{\sqrt{c}} y-x\right)\right] p_{j, \varepsilon}^{*}\left(\frac{2}{\sqrt{c}} y-x\right) f(y) d y
\end{aligned}
$$


and

$$
f_{n N}(x)=T^{N-n} \exp \left[\beta 2^{N a / 2} h\left(\sigma^{\prime}\right) x\right] .
$$

It is noteworthy that in such a case the random variables $\xi_{m}$ form a stationary Markov chain. In the theorem we consider $\sigma^{\prime}=0$ so $\exp \left[\beta 2^{N a / 2} h\left(\sigma^{\prime}\right) x\right] \equiv 1$ and $f_{n N}(x)=T^{N-n} 1$.

The operator

$$
S=\exp \left(-\alpha x^{2}\right) T \exp \left(\alpha x^{2}\right), \quad \alpha>0,
$$

is equivalent to $T$ and

$$
f_{n N}(x)=\exp \left(\alpha x^{2}\right) S^{N-n} \exp \left(-\alpha x^{2}\right) .
$$

The kernel of the operator $S$ has the form

$$
\begin{aligned}
S(x, y)= & \exp \left[\frac{c}{4} x\left(\frac{2}{\sqrt{c}} y-x\right)-\alpha x^{2}+\alpha y^{2}\right] p_{j, \varepsilon}^{*}\left(\frac{2}{\sqrt{c}} y-x\right) \\
= & \exp \left[\frac{c}{4} x\left(\frac{2}{\sqrt{c}} y-x\right)-\alpha x^{2}+\alpha y^{2}\right. \\
& \left.-\frac{c}{4(2-c)}\left(\frac{2}{\sqrt{c}} y-x\right)^{2}-\varphi_{j, \varepsilon}^{*}\left(\frac{2}{\sqrt{c}} y-x\right)\right] .
\end{aligned}
$$

Direct calculations show that for $\alpha=\frac{c^{2}-3 c+4}{8(2-c)}>0$,

$$
\begin{array}{r}
S(x, y)=S_{0}(x, y) \exp \left[-\varphi_{j, \varepsilon}^{*}\left(\frac{2}{\sqrt{c}} y-x\right)\right], \\
S_{0}(x, y)=\exp \left[-A(x-y)^{2}-B x^{2}-B y^{2}\right],
\end{array}
$$

where

$$
A=\frac{(4-c) \sqrt{c}}{(2-c) 4}>0, B=\frac{4-c}{(2-c) 8}(\sqrt{c}-1)^{2}>0 .
$$

The operator $S_{0}$ with the kernel $S_{0}(x, y)$ is a compact self-adjoint operator in $L^{2}\left(\mathbb{R}^{1}\right)$. Its eigenfunctions have the form:

$$
e_{j}^{(0)}(x)=\exp \left(-\gamma x^{2}\right) H_{j}(\sqrt{2 \gamma} x)
$$

where $\gamma=\sqrt{B^{2}+2 A B}=\frac{(4-c)(c-1)}{8(2-c)}$ and $H_{j}(t)$ are the Hermite polynomials, and the corresponding eigenvalues:

$$
\lambda_{j}^{(0)}=\frac{\lambda_{0}^{(0)}}{c^{j / 2}}
$$

Let $L_{0} \subset L^{2}\left(\mathbb{R}^{1}\right)$ be the space orthogonal to $e_{0}^{(0)}(x)$. Then for $f \in L_{0}$

$$
\left\|S_{0} f\right\| \leqq \frac{\lambda_{0}^{(0)}}{\sqrt{c}}\|f\|,
$$

where $\|\cdot\|$ is the $L^{2}$-norm. 
Using perturbation theory arguments one can prove that for the operator $S$ an eigenfunction $e_{0}(x)$ and a supplementary subspace $L \subset L^{2}\left(\mathbb{R}^{1}\right)$ also exist such that

where

$$
\begin{gathered}
\quad S e_{0}=\lambda_{0} e_{0}, \\
\|S f\| \leqq \frac{\lambda_{0}}{\sqrt{c}-\delta}\|f\|, \quad f \in L,
\end{gathered}
$$

$$
\delta,\left(\lambda_{0}^{(0)}-\lambda_{0}\right),\left\|e_{0}^{(0)}-e_{0}\right\|=O(\varepsilon)
$$

(see $[2,3,9]$ ). The subspace $L$ is orthogonal to the first eigenfunction $e_{0}^{\prime}$ of the adjoint operator $S^{\prime}$ and

$$
\left\|e_{0}^{(0)}-e_{0}^{\prime}\right\|=O(\varepsilon)
$$

Let us write:

$$
\begin{gathered}
f(x)=\mu e_{0}(x)+f^{\prime}(x), \\
\mu=\left(f, e_{0}^{\prime}\right), f^{\prime} \in L .
\end{gathered}
$$

Then

$$
S^{N-n} f=\mu \lambda_{0}^{N-n} e_{0}+f_{N-n}^{\prime}
$$

where

$$
\left\|f_{N-n}^{\prime}\right\| \leqq\left(\frac{\lambda_{0}}{\sqrt{c}-\delta}\right)^{N-n}\left\|f^{\prime}\right\|
$$

so if $\mu \neq 0$

$$
\left(\mu \lambda_{0}^{N-n}\right)^{-1} S^{N-n} f=e_{0}+O\left(\left(\frac{1}{\sqrt{c}-\delta}\right)^{N-n} \frac{\left\|f^{\prime}\right\|}{\mu}\right) .
$$

To estimate $\mu$ we use Eq. (3.14):

$$
\mu=\left(f, e_{0}^{\prime}\right)=\left(f, e_{0}^{(0)}\right)+O(\varepsilon)=\sqrt[4]{\frac{\gamma}{\pi}} \int f(x) \exp \left(-\gamma x^{2}\right) d x+O(\varepsilon) .
$$

In our case

$$
f(x)=\exp \left(-\alpha x^{2}\right)
$$

and for small $\varepsilon$ the quantity $\frac{\left\|f^{\prime}\right\|}{\mu}$ is positive and uniformly bounded in $\varepsilon$. Moreover we can suppose that $(\sqrt{c}-\delta)^{-1}<\varrho<1$. So

$$
S^{N-n} f(x)=L_{N-n}^{-1}\left[e_{0}(x)+O\left(Q^{N-n}\right)\right],
$$

where $L_{N-n}^{-1}$ is a normalizing factor. Here the estimate of the remainder term is in $L^{2}$-norm. Let us note however that the operator $S$ is continuous from $L^{2}\left(\mathbb{R}^{1}\right)$ to $\mathscr{S}\left(\mathbb{R}^{1}\right)$, so $e_{0}(x) \in \mathscr{S}\left(\mathbb{R}^{1}\right)$ and the formula (3.20) is also valid for the sequence of norms defining the topology in $\mathscr{S}\left(\mathbb{R}^{1}\right)$. 
Thus it is proved that

$$
f_{n N}(x) / f_{n N}(0)=\exp \left(\alpha x^{2}\right)\left[e_{0}(x)+O\left(\varrho^{N-n}\right)\right] /\left[e_{0}(0)+O\left(\varrho^{N-n}\right)\right],
$$

where $0<\varrho<1$, hence

$$
f_{n N}(x) / f_{n N}(0)_{N \rightarrow \infty} \exp \left(\alpha x^{2}\right) e_{0}(x) / e_{0}(0) .
$$

As the distribution $\mu_{n}\left(d \sigma \mid v_{j, \varepsilon}^{*}, 0\right)$ has a density decreasing at infinity as $\prod_{i \in V_{n 0}}\left\{C_{1} \exp \left[-C_{2} \varepsilon \sigma(i)^{2 j}\right]\right\}$, i.e. faster than any Gaussian density, then (3.8) and (3.21) imply the convergence of the distributions $\mu_{n N}\left(d \sigma \mid v_{j, \varepsilon}^{*}, \sigma^{\prime}\right)$ to the limit $L^{-1} \exp \left(\alpha \xi_{n}^{2}\right) e_{0}\left(\xi_{n}\right) \mu_{n}\left(d \sigma \mid v_{j, \varepsilon}^{*}, 0\right)$ when $N \rightarrow \infty$. Theorem 2 is proved.

As a final remark to this proof we would like to draw attention to the fact that the first two eigenfunctions and eigenvalues of the adjoint operator $S^{\prime}$ can be found explicitly. Namely,

$$
\begin{aligned}
S^{\prime} & =\exp \left(\alpha x^{2}\right) T^{\prime} \exp \left(-\alpha x^{2}\right), \\
T^{\prime}: f(x) & \rightarrow \int \exp \left[\frac{c}{4} y\left(\frac{2}{\sqrt{c}} x-y\right)\right] p_{j, \varepsilon}^{*}\left(\frac{2}{\sqrt{c}} x-y\right) f(y) d y \\
& =\iint_{\frac{x_{1}+x_{2}}{2^{a / 2}}=x} \exp \left(\frac{c}{4} x_{1} x_{2}\right) p_{j, \varepsilon}^{*}\left(x_{1}\right) f\left(x_{2}\right) d x_{1} d x_{2} .
\end{aligned}
$$

As $p_{j, \varepsilon}^{*}(x)$ satisfies the fixed point equation (2.2) then

$$
T^{\prime} p_{j, \varepsilon}^{*}=\lambda_{0} p_{j, \varepsilon}^{*},
$$

where

$$
\lambda_{0}=2 \iint \exp \left(\frac{c}{4} x_{1} x_{2}\right) p_{j, \varepsilon}^{*}\left(x_{1}\right) p_{j, \varepsilon}^{*}\left(x_{2}\right) d x_{1} d x_{2} .
$$

Thus $p_{j, \varepsilon}^{*}$ is an eigenfunction of the operator $T^{\prime}$ and so

$$
e_{0}^{\prime}(x)=\exp \left(\alpha x^{2}\right) p_{j, \varepsilon}^{*}(x)
$$

is an eigenfunction of the operator $S^{\prime}$.

Differentiating in $x$ the fixed point equation for the function $\exp \left[-\varphi_{j, \varepsilon}^{*}(x)\right]$ one comes to the equality

$$
T^{\prime} p_{1}(x)=\lambda_{1} p_{1}(x)
$$

where

$$
p_{1}(x)=\exp \left(-a_{0} x^{2}\right) \frac{d}{d x} \exp \left[-\varphi_{j, \varepsilon}^{*}(x)\right]
$$

and $\lambda_{1}=\frac{\lambda_{0}}{\sqrt{c}}$. So

$$
S^{\prime} e_{1}^{\prime}(x)=\lambda_{1} e_{1}^{\prime}(x)
$$


where

$$
e_{1}^{\prime}(x)=\exp \left(\alpha x^{2}\right) p_{1}(x)=\exp \left[\left(\alpha-a_{0}\right) x^{2}\right] \frac{d}{d x} \exp \left[-\varphi_{j, \varepsilon}^{*}(x)\right]
$$

$\lambda_{0}$ is the largest eigenvalue of the operator $S^{\prime}, \lambda_{1}$ is the second one. It can be noted that $e_{0}^{\prime}(x)$ is an even function while $e_{1}^{\prime}(x)$ is an odd one. The two largest eigenvalues of the operator $S$ are of course the same: $\lambda_{0}$ and $\lambda_{1}=\frac{\lambda_{0}}{\sqrt{c}}$.

Proof of Theorem 3. Following the proof of Theorem 2 we have to establish only the convergence of the functions $g_{n N}(x)=f_{n N}(x) / f_{n N}(0)$ when $N \rightarrow \infty$, where

$$
f_{n N}(x)=T^{N-n} \exp \left(2^{N a / 2} h\left(\sigma_{N}^{\prime}\right) x\right) .
$$

As in the proof of Theorem 2 we write:

$$
f_{n N}(x)=\exp \left(\alpha x^{2}\right) S^{N-n} f(x),
$$

where

$$
f(x)=\exp \left(-\alpha x^{2}+2^{N a / 2} h\left(\sigma_{N}^{\prime}\right) x\right)=C_{N} \exp \left[-\alpha\left(x-\frac{2^{N a / 2} h\left(\sigma_{N}^{\prime}\right)}{2 \alpha}\right)^{2}\right] .
$$

The previous proof, based in fact on a variant of the Perron-Frobenius theorem, is inapplicable here in view of the following circumstance: For large values of $2^{N a / 2} h\left(\sigma_{N}^{\prime}\right)$ the function $f(x)$ is large only far from the origin, in the neighbourhood of the point $2^{N a / 2} h\left(\sigma_{N}^{\prime}\right) / 2 \alpha$, and so the quantity $\left(f, e_{0}^{\prime}\right) /\left\|f^{\prime}\right\|$ entering the right hand side of the estimate (3.18) is extremely small: $\left(f, e_{0}^{\prime}\right) /\left\|f^{\prime}\right\| \sim \exp \left[-2^{N a / 2} h\left(\sigma_{N}^{\prime}\right) / 2 \alpha\right]$. Therefore the estimate (3.18) becomes of little use. In view of this we change the scheme of our considerations. We divide our proof into two stages. At the first one we prove an approximate equality

$$
S^{k} f(x) / S^{k} f(0) \approx \exp \left[-\alpha x^{2}+2^{(N-k) a / 2} h\left(\sigma_{N}^{\prime}\right) x\right]
$$

for all $k$ such that $2^{(N-k) a / 2}\left|h\left(\sigma_{N}^{\prime}\right)\right|>\varepsilon^{-10}$. In particular one can find such a $k_{0}$ for which $\varepsilon^{-10}<2^{\left(N-k_{0}\right) a / 2}\left|h\left(\sigma_{N}^{\prime}\right)\right|<\varepsilon^{-11}$ and the approximate equality (3.23) is true. From this equality we derive the bound

$$
\frac{\left(S^{k_{0}} f, e_{0}^{\prime}\right)}{\left\|S^{k_{0}} f\right\|}>\tau>0
$$

where $\tau$ depends on $\varepsilon$ but does not depend on $N, h\left(\sigma_{N}^{\prime}\right)$ and $k_{0}$. As $S^{N-n} f=S^{N-k_{0}-n}\left(S^{k_{0}} f\right), h\left(\sigma_{N}^{\prime}\right) \rightarrow 0$ and $2^{\left(N-k_{0}\right) a / 2}\left|h\left(\sigma_{N}^{\prime}\right)\right|>\varepsilon^{-10}$, then $N-k_{0 N} \rightarrow \infty \infty$. It allows us at the second stage of our proof to obtain the convergence

$$
\frac{S^{N-k_{0}-n}\left(S^{k_{0}} f\right)(x)}{S^{N-n} f(0)}{ }_{N \rightarrow \infty} e_{0}(x)
$$

which finishes the proof of the theorem.

To specify the approximate equality (3.23) we write

$$
S^{k} f(x)=\exp \left[-\varphi_{k}(x)\right]
$$


Our reasoning rests on obtaining suitable bounds for the second derivative $\varphi_{k}^{\prime \prime}(x)$. It turns out that the latter takes values close to $2 \alpha$ everywhere except on a sequence of segments. Let

$$
\xi=\left(\sqrt{c}-\frac{c}{2}\right) A-\frac{c}{2} B-\sqrt{c} \alpha .
$$

A direct calculation shows that $\xi>0$ if $1<c<\sqrt{2}, c=2^{2-a}$. Let us consider for $k \geqq 1$ the points

$$
x_{k i}=\xi^{-1} h_{k} 2^{i a}, i=0,1, \ldots, k-1 \text {, }
$$

where

$$
h_{k}=2^{(N-k) a / 2} h\left(\sigma_{N}^{\prime}\right)
$$

and the segments

$$
\Delta_{k i}=\left\{x|| x / x_{k i}-1 \mid \leqq \delta\left(1-\left(\frac{7}{8}\right)^{i+1}\right)\right\}, i=0,1, \ldots, k-1,
$$

where $\delta=\frac{0.01}{2 \alpha}$ (see Fig. 1$)$.

Fig. 1

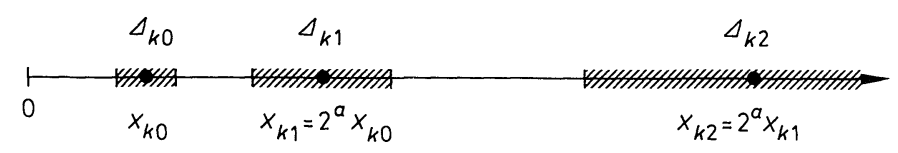

Denote $\Delta_{k}=\bigcup_{i=0}^{k-1} \Delta_{k i}$. The following lemma is central in our considerations.

Lemma 3.1. Assume that $\left|h_{k}\right|>\varepsilon^{-10}$, where $h_{k}=2^{(N-k) a / 2} h\left(\sigma_{N}^{\prime}\right)$. Then the following estimates hold:

$$
\begin{aligned}
& \left|\varphi_{k}^{\prime}(0)-h_{k}\right|<\left|h_{k}\right|^{1 / 2}, \\
& \left|\varphi_{k}^{\prime \prime}(x)-2 \alpha\right|<\left|h_{k}\right|^{-1 / 3} \quad \text { if } \quad x \notin \Delta_{k}, \\
& \left|\varphi_{k}^{\prime \prime}(x)-2 \alpha\right|<b_{i} \quad \text { if } \quad x \in \Delta_{k i},
\end{aligned}
$$

where $b_{0}=\frac{7}{4} \alpha$ and $b_{i+1}=\frac{1}{2}\left(b_{i}+\left|h_{k}\right|^{-1 / 3}\right), i=0,1, \ldots, k-2$.

Proof of the lemma will be given below and now we finish the proof of the theorem.

By (3.28), (3.29), $\varphi_{k}^{\prime \prime}(x)>\alpha / 4>0$, so $\varphi_{k}^{\prime}(x)$ is a monotonous function of $x$ and the equation

$$
\varphi_{k}^{\prime}(m)=0
$$

has an unique solution. By (3.27)-(3.29) this solution lies in the interval $\left[0,5 h_{k} / \alpha\right]$. Moreover

so

$$
2 \alpha(x-m)^{2} \geqq \varphi_{k}(x)-\varphi_{k}(m) \geqq \frac{\alpha}{8}(x-m)^{2},
$$

$$
\begin{aligned}
\left\|S^{k} f(x)\right\| & \leqq S^{k} f(m)\left\{\int_{-\infty}^{\infty} \exp \left[-\frac{\alpha}{4}(x-m)^{2}\right] d x\right\}^{1 / 2} \\
& \leqq C_{0} S^{k} f(m)
\end{aligned}
$$


and

$$
\left(S^{k} f, e_{0}^{\prime}\right) \geqq S^{k} f(m) \int_{-\infty}^{\infty} e_{0}^{\prime}(x) \exp \left[-2 \alpha(x-m)^{2}\right] d x .
$$

If $\left|h_{k_{0}}\right|=\left|2^{\left(N-k_{0}\right) a / 2} h\left(\sigma_{N}^{\prime}\right)\right|<\varepsilon^{-11}$, then for $k=k_{0}$ the point $m$ lies in the interval $\left[0,5 \varepsilon^{-11} / \alpha\right]$. As $e_{0}^{\prime}(x)$ is a strictly positive function [see (3.22)] then for some $C_{1}>0$ which does not depend on $N, h\left(\sigma_{N}^{\prime}\right)$ and $k_{0}$,

so

$$
\int_{-\infty}^{\infty} e_{0}^{\prime}(x) \exp \left[-2 \alpha(x-m)^{2}\right] d x>C_{1}
$$

$$
\frac{\left(S^{k_{0}} f, e_{0}^{\prime}\right)}{\left\|S^{k_{0}} f\right\|} \geqq \frac{C_{1} S^{k} f(m)}{C_{0} S^{k} f(m)}=\frac{C_{1}}{C_{0}}=\tau>0
$$

Now using the same method as in the proof of Theorem 2 we obtain (3.24) and next the statement of Theorem 3. It remains to prove the lemma above.

Proof of Lemma 3.1. The proof is by induction in $k$. For $k=0$ the statement of the lemma is obvious because

$$
\varphi_{0}(x)=\varphi_{0}(0)-\alpha x^{2}+2^{N a / 2} h\left(\sigma_{N}^{\prime}\right) x=\varphi_{0}(0)-\alpha x^{2}+h_{0} x .
$$

Assume now that $\left|h_{k+1}\right|>\varepsilon^{-10}$ and the statement of the lemma is true for $\varphi_{k}(x)$. Denote for brevity

$$
\begin{aligned}
\varphi(x) & =\varphi_{k}(x), \psi(x)=\varphi_{k+1}(x), h=h_{k}, \Delta=\Delta_{k+1,0} \\
& =\left\{x|| x 2^{a / 2} / h-1 \mid<\delta / 8\right\}, \varphi^{*}(x)=\varphi_{j, \varepsilon}^{*}\left(2^{a / 2} x\right) .
\end{aligned}
$$

Without loss of generality we may assume that $h>0$. Let

$$
\Phi(x, y)=A(x-y)^{2}+B x^{2}+B y^{2}+\varphi^{*}\left(y-\frac{x \sqrt{c}}{2}\right)+\varphi(y) .
$$

Then

$$
\psi(x)=-\ln \int_{-\infty}^{\infty} \exp (-\Phi(x, y)) d y .
$$

For fixed $x$ a minimum point $m=m(x)$ of the function $\Phi(x, y)$ in $y$ is defined by the equation

$$
\left.\frac{\partial \Phi}{\partial y}\right|_{y=m}=-2 A x+2(A+B) m+\varphi^{* \prime}\left(m-\frac{x \sqrt{c}}{2}\right)+\varphi^{\prime}(m)=0 .
$$

As

$$
\begin{aligned}
\frac{\partial^{2} \Phi}{\partial y^{2}} & =2(A+B)+\varphi^{* \prime \prime}\left(y-\frac{x \sqrt{c}}{2}\right)+\varphi^{\prime \prime}(y) \\
& >2(A+B)-C_{0} \varepsilon+\frac{\alpha}{4}>2 A>0
\end{aligned}
$$

the function $\frac{\partial \Phi}{\partial y}$ is monotonous in $y$ and Eq. (3.32) has a unique solution. It is extremely important for us that for large $|x|$ this solution lies near the point $\frac{x \sqrt{c}}{2}$. 
This fact is due to the fast increase of the function $\varphi^{*^{\prime}}(t)$. Namely using the estimate

one obtains easily that

$$
\varphi^{*^{\prime}}(t)>C_{1} \varepsilon\left(|t|^{3}-1\right)
$$

where

$$
m=\frac{x \sqrt{c}}{2}+\tilde{m}
$$

$$
|\tilde{m}|<C_{2}\left(\frac{|x|+|h|}{\varepsilon}\right)^{1 / 3}
$$

It is noteworthy that this estimate implies two properties important for what follows:

(i)

$$
\begin{gathered}
\mathbb{R}^{1} \bigcup_{l \neq i-1} \Delta_{k l} \supset m\left(\Delta_{k+1, i}\right) \supset \Delta_{k, i-1}, i=1, \ldots, k, \\
m\left(\mathbb{R}^{1} \bigcup_{i=1}^{k} \Delta_{k+1, i}\right) \subset \mathbb{R}^{1} \bigcup_{i=0}^{k-1} \Delta_{k i}=\mathbb{R}^{1} \backslash \Delta_{k},
\end{gathered}
$$

where $m(X)$ is the image of a set $X \subset \mathbb{R}^{1}$ under the map $x \rightarrow m(x)$. Roughly speaking $m$ maps $\Delta_{k+1, i}$ into $\Delta_{k, i-1}$ for $i=1,2, \ldots, k$.

Furthermore, (3.33) implies that

$$
\begin{gathered}
\Phi(x, y) \geqq \Phi(x, m)+A(y-m)^{2}, \\
\exp (-\Phi(x, y)) \leqq \exp \left(-\Phi(x, m)-A(y-m)^{2}\right),
\end{gathered}
$$

so the main contribution to $\psi(x)$ is given by $y$ 's from a $O(1)$-neighbourhood of the point $m$. For $x \notin \Delta$, the estimate (3.33) can be improved on account of the term $\varphi^{* \prime \prime}\left(y-\frac{x \sqrt{c}}{2}\right)$. Namely we shall show now that if $|y-m|<h^{1 /\left(a^{\prime}-1\right)}$ and $x \notin \Delta$, $a^{\prime}=\frac{1}{2-a}$, then

$$
\frac{\partial^{2} \Phi(x, y)}{\partial y^{2}}>2 A+C_{2} \varepsilon h^{2 / 3}>2 A+h^{1 / 2} .
$$

This means that the main contribution to $\psi(x)$ is given by $y$ 's from a $O\left(h^{-1 / 4}\right)$ neighbourhood of the point $m$.

To prove the estimate (3.37) we remark that the conditions (3.27)-(3.29) imply that

where

$$
\varphi^{\prime}(y)=h+2 \alpha y+r(y),
$$

where $\Omega=\left\{x \mid x<x_{k 0}(1-\delta)=\xi^{-1} h(1-\delta)\right\}$ (see Fig. 2).

Fig. 2

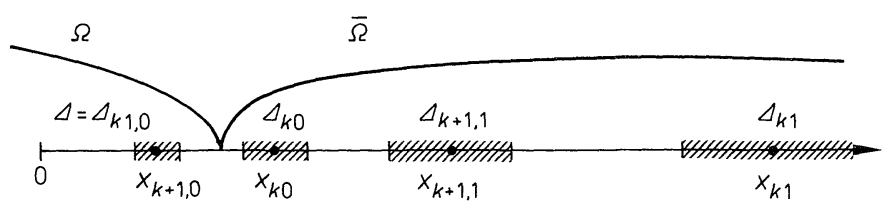


Substitute (3.34) and (3.38) into (3.32):

$$
\begin{aligned}
& -2 A x+2(A+B)\left(\frac{\sqrt{c}}{2} x+\tilde{m}\right)+h+2 \alpha\left(\frac{\sqrt{c}}{2} x+\tilde{m}\right) \\
& +r(m)+\varphi^{* \prime}\left(m-\frac{x \sqrt{c}}{2}\right)=0,
\end{aligned}
$$

or

$$
\frac{2}{\sqrt{c}} \xi x-h-\eta \tilde{m}-r(m)=\varphi^{* \prime}\left(m-\frac{x \sqrt{c}}{2}\right)
$$

where $\xi$ was defined in (3.26) and $\eta=2(A+B+\alpha)$. The meaning of introducing the segment $\Delta=\Delta_{k+1,0}$ is that for $x \notin \Delta,\left|\frac{2}{\sqrt{c}} \xi x-h\right|>\delta h$. It allows us to infer from (3.39), (3.34'), and (3.38') that

$$
\left|\varphi^{* \prime}\left(m-\frac{x \sqrt{c}}{2}\right)\right|>\frac{\delta h}{2}
$$

and hence that [see (2.6), here $a^{\prime}=(2-a)^{-1}$ ]

$$
\left|m-\frac{x \sqrt{c}}{2}\right|>C\left(\varepsilon^{-1} h\right)^{1 /\left(a^{\prime}-1\right)}
$$

where $C>0$ is a constant. Now if $|y-m|<h^{1 /\left(a^{\prime}-1\right)}$, then

$$
\left|y-\frac{x \sqrt{c}}{2}\right| \geqq\left|m-\frac{x \sqrt{c}}{2}\right|-|y-m|>C\left(\varepsilon^{-1} h\right)^{1 /\left(a^{\prime}-1\right)}-h^{1 /\left(a^{\prime}-1\right)}>C h^{1 /\left(a^{\prime}-1\right)},
$$

so

$$
\varphi^{\prime \prime}\left(y-\frac{x \sqrt{c}}{2}\right)>C_{1} \varepsilon\left|y-\frac{x \sqrt{c}}{2}\right|^{a^{\prime}-2}>C_{2} \varepsilon h^{\frac{a^{\prime}-2}{a^{\prime}-1}}>C_{2} \varepsilon h^{2 / 3}
$$

which proves (3.37). We would like to note here that the estimate (3.41) shows that though the point $m$ lies relatively near $\frac{x \sqrt{c}}{2}$ as we have seen in $\left(3.34^{\prime}\right)$, the absolute distance between them is not too small unless $x \in \Delta$.

By (3.31) we have directly that

$$
\begin{aligned}
\psi^{\prime}(x) & =\left\langle\frac{\partial \Phi}{\partial x}\right\rangle \\
\psi^{\prime \prime}(x) & =\left\langle\frac{\partial^{2} \Phi}{\partial x^{2}}\right\rangle-\left\langle\left(\frac{\partial \Phi}{\partial x}\right)^{2}\right\rangle+\left\langle\frac{\partial \Phi}{\partial x}\right\rangle^{2} \\
& =\left\langle\frac{\partial^{2} \Phi}{\partial x^{2}}\right\rangle-\left\langle\left(\frac{\partial \Phi}{\partial x}-\left\langle\frac{\partial \Phi}{\partial x}\right\rangle\right)^{2}\right\rangle,
\end{aligned}
$$


where we use the notation

$$
\langle f\rangle \equiv \frac{\int_{-\infty}^{\infty} f(x, y) \exp (-\Phi(x, y)) d y}{\int_{-\infty}^{\infty} \exp (-\Phi(x, y)) d y} .
$$

Let us note some simple properties of $\langle\cdot\rangle$ :

(i) $\langle 1\rangle=1,|\langle f\rangle| \leqq \sup _{y}|f|$,

(ii) $\left\langle\frac{\partial \Phi}{\partial y}\right\rangle=0,\left\langle f \frac{\partial \Phi}{\partial y}\right\rangle=\left\langle f^{\prime}\right\rangle$, $\left\langle\frac{\partial^{2} \Phi}{\partial y^{2}}-\left(\frac{\partial \Phi}{\partial y}\right)^{2}\right\rangle=0$

(iii) if $x \notin \Delta$ then $\left\langle|x-m|^{k}\right\rangle \leqq C_{k} h^{-k / 4}, k=1,2, \ldots$.

The property (i) is obvious, (ii) is proved by integration by parts, and (iii) is a consequence of the inequalities (3.33), (3.37).

Now perform:

$$
\frac{\partial \Phi}{\partial x}=2(A+B) x-2 A y-\frac{\sqrt{c}}{2} \varphi^{*^{\prime}}\left(y-\frac{x \sqrt{c}}{2}\right),
$$

and by (3.32)

$$
\varphi^{* \prime}\left(y-\frac{x \sqrt{c}}{2}\right)=\frac{\partial \Phi}{\partial y}+2 A x-2(A+B) y-\varphi^{\prime}(y)
$$

so

$$
\frac{\partial \Phi}{\partial x}=\lambda x+\mu y-\frac{\sqrt{c}}{2} \frac{\partial \Phi}{\partial y}+\frac{\sqrt{c}}{2} \varphi^{\prime}(y),
$$

where $\lambda=2 A+2 B-\sqrt{c} A, \mu=-2 A+\sqrt{c} A+\sqrt{c} B$. Thus

$$
\begin{aligned}
\left\langle\frac{\partial \Phi}{\partial x}\right\rangle & =\left\langle\lambda x+\mu y+\frac{\sqrt{c}}{2} \varphi^{\prime}(y)\right\rangle \\
& =\lambda x+\mu m+\frac{\sqrt{c}}{2} \varphi^{\prime}(m)+\langle\theta\rangle,
\end{aligned}
$$

where

$$
\theta=\mu(y-m)+\frac{\sqrt{c}}{2}\left(\varphi^{\prime}(y)-\varphi^{\prime}(m)\right)
$$

Analogously one obtains the formula

$$
\begin{aligned}
\left\langle\frac{\partial^{2} \Phi}{\partial x^{2}}\right\rangle & =v+\frac{c}{4} \varphi^{\prime \prime}(m)-\left\langle\theta^{2}\right\rangle+\langle\theta\rangle^{2} \\
& =v+\frac{c}{4} \varphi^{\prime \prime}(m)-\left\langle(\theta-\langle\theta\rangle)^{2}\right\rangle
\end{aligned}
$$


where

$$
\nu=2\left[\left(1-\frac{\sqrt{c}}{2}\right)^{2} A+\left(1+\frac{c}{4} B\right)\right] .
$$

Using the identities (3.44), (3.46) we shall derive now the desired relations (3.27)-(3.29) for the function $\psi(x)$. As these relations are fulfilled for $\varphi(x)$, then

$$
\left|\varphi^{\prime}(y)-\varphi^{\prime}(m)\right| \leqq 4 \alpha|y-m|,
$$

so by the property (iii)

$$
|\langle\theta\rangle| \leqq\langle|\mu(y-m)|\rangle+\langle|2 \alpha \sqrt{c}(y-m)|\rangle \leqq C h^{-1 / 4}
$$

if $x \notin \Delta$ and similarly

Therefore

$$
\left\langle\theta^{2}\right\rangle \leqq C_{0} h^{-1 / 2}
$$

$$
\psi^{\prime \prime}(x)=\left\langle\frac{\partial^{2} \Phi}{\partial x^{2}}\right\rangle=\nu+\frac{c}{4} \varphi^{\prime \prime}(m)+\varrho(x)
$$

where

$$
|\varrho(x)|<C_{1} h^{-1 / 2} .
$$

Next, if $x \notin \Delta_{k+1}=\Delta \cup\left(\bigcup_{i=1}^{k} \Delta_{k+1, i}\right)$ then by (3.36), $m=m(x) \in \Delta_{k}$, so by (3.28),

$$
\varphi^{\prime \prime}(m)=2 \alpha+\varrho_{1}(x),
$$

where $\left|\varrho_{1}(x)\right|<h^{-1 / 3}$. A direct calculation gives that

$$
v+\frac{c \alpha}{2}=2 \alpha
$$

Therefore

$$
\begin{aligned}
\left|\psi^{\prime \prime}(x)-2 \alpha\right| & =\left|\frac{c}{4} \varrho_{1}(x)+\varrho(x)\right|<\frac{c}{4} h^{-1 / 3}+C_{1} h^{-1 / 2} \\
& <\left(\frac{\sqrt{c}}{2} h\right)^{-1 / 3}=h_{k+1}^{-1 / 3} .
\end{aligned}
$$

Thus we have established (3.28) for $\psi(x)=\varphi_{k+1}(x)$. Furthermore, if $x \in \Delta_{k+1, i}$, $i=1, \ldots, k$, then by (3.35), $m \notin \bigcup_{l \neq i-1} A_{k l}$ so by (3.28), (3.29), (3.49),

$$
\left|\varrho_{1}(x)\right|=\left|\varphi_{k}^{\prime \prime}(x)-2 \alpha\right| \leqq b_{i}
$$

and

$$
\begin{aligned}
\left|\psi^{\prime \prime}(x)-2 \alpha\right| & =\left|\frac{c}{4} \varrho_{1}(x)+\varrho(x)\right| \leqq \frac{c}{4} b_{i}+C_{1} h^{-1 / 2} \\
& \leqq \frac{1}{2}\left(b_{i}+h_{k+1}^{-1 / 3}\right),
\end{aligned}
$$

which proves (3.29) for $\psi(x)=\varphi_{k+1}(x)$ if $x \in \Delta_{k+1, i}, i=1, \ldots, k$. 
To prove (3.27) for $\psi(x)$ we note that by (3.44)

$$
\begin{aligned}
\psi^{\prime}(0) & =\left\langle\left.\frac{\partial \Phi}{\partial x}\right|_{x=0}\right\rangle=\mu m+\frac{\sqrt{c}}{2} \varphi^{\prime}(m)+\langle\theta\rangle \\
& =\frac{\sqrt{c}}{2} \varphi^{\prime}(0)+\varrho_{2},
\end{aligned}
$$

where

$$
\begin{aligned}
& \left|\varrho_{2}\right| \leqq|\mu m|+\frac{\sqrt{c}}{2} 4 \alpha|m|+C h^{-1 / 4}<C_{1}\left|\varepsilon^{-1} h\right|^{1 / 3} \\
& +C h^{-1 / 4}<0.1 h^{1 / 2} .
\end{aligned}
$$

So by (3.27)

$$
\begin{aligned}
\left|\psi^{\prime}(0)-\frac{\sqrt{c}}{2} h\right|= & \left|\frac{\sqrt{c}}{2}\left(\varphi^{\prime}(0)-h\right)+\varrho_{2}\right|<\frac{\sqrt{c}}{2} h^{1 / 2} \\
& +0.1 h^{1 / 2}<\left(\frac{\sqrt{c}}{2} h\right)^{1 / 2}=h_{k+1}^{1 / 2},
\end{aligned}
$$

as required.

It remains to prove (3.29) for $x \in \Delta=\Delta_{k+1,0}$. In this case $m=m(x) \notin \Delta_{k}$ so (3.28) holds. Next, the upper bound follows immediately from (3.46) as $\left\langle(\theta-\langle\theta\rangle)^{2}\right\rangle \geqq 0$. To prove the lower bound we have to estimate $\left\langle\theta^{2}\right\rangle$. One easily has from (2.6) and (3.27) that if $x \in \Delta$ and $|y-m(x)|<|\ln \varepsilon|$ then

$$
\begin{aligned}
\frac{\partial^{2} \Phi(x, y)}{\partial y^{2}} & =2(A+B)+\varphi^{* \prime \prime}\left(y-\frac{x \sqrt{c}}{2}\right)+\varphi^{\prime \prime}(y) \\
& =\left[2(A+B)+\varphi^{* \prime \prime}\left(m-\frac{x \sqrt{c}}{2}\right)+\varphi^{\prime \prime}(m)\right](1+\varrho(x, y)) \\
& =\left.\frac{\partial^{2} \Phi}{\partial y^{2}}\right|_{y=m}(1+\varrho(x, y)),
\end{aligned}
$$

where $|\varrho(x, y)|<\varepsilon^{1 / 3}$. Therefore

$$
\left|\left\langle(y-m)^{k}\right\rangle_{0}-\left\langle(y-m)^{k}\right\rangle\right| \leqq C_{k} \varepsilon^{1 / 3},
$$

where

$$
\begin{gathered}
\langle f\rangle_{0}=\frac{\int_{-\infty}^{\infty} f(x, y) \exp \left(-\Phi_{0}(x, y)\right) d y}{\int_{-\infty}^{\infty} \exp \left(-\Phi_{0}(x, y)\right) d y}, \\
\Phi_{0}(x, y)=\Phi(x, m)+\left.\frac{1}{2} \frac{\partial^{2} \Phi}{\partial y^{2}}\right|_{y=m}(y-m)^{2} .
\end{gathered}
$$


So it is sufficient to estimate $\left\langle\theta^{2}\right\rangle$. Furthermore, for $|y-m|<|\ln \varepsilon|$,

$$
\begin{aligned}
\theta & =\mu(y-m)+\frac{\sqrt{c}}{2}\left(\varphi^{\prime}(y)-\varphi^{\prime}(m)\right)=(\mu+\sqrt{c} \alpha)(y-m)+R(y) \\
& =\kappa(y-m)+R(y),
\end{aligned}
$$

where $\kappa=\mu+\sqrt{c} \alpha,|R(y)| \leqq C|y-m|^{2} h^{-1 / 3}$, so

$$
\begin{gathered}
\left\langle\theta^{2}\right\rangle_{0}=\kappa^{2}\left\langle(y-m)^{2}\right\rangle_{0}+R_{1} \\
=\kappa^{2}\left(\left.\frac{\partial^{2} \Phi}{\partial y^{2}}\right|_{y=m}\right)^{-1}+R_{1},
\end{gathered}
$$

where $\left|R_{1}\right|<\varepsilon^{1 / 3}$. This gives an approximate expression for $\left\langle\theta^{2}\right\rangle_{0}$ and hence for $\left\langle\theta^{2}\right\rangle$. Substituting it in (3.46) we come to the desired lower bound of $\psi^{\prime \prime}(x)$.

The lemma is proved.

Proof of Theorem 4. By the previous theorem the limit $\mu_{n \infty}(d \sigma \mid \beta$, $\left.v_{j, \varepsilon, \beta}^{*}\right)=\lim _{N \rightarrow \infty} \mu_{n N}\left(d \sigma \mid \beta, v_{j, \varepsilon, \beta}^{*}, \sigma_{N}^{\prime}\right)$ exists and is unique for any sequence $\sigma_{N}^{\prime}$ such that $h\left(\sigma_{N}^{\prime}\right)_{N \rightarrow \infty} 0$. Moreover one can see easily from the proof of this theorem that the convergence is uniform in the following sense: For any Borel set $M \subset \sum\left(V_{n 0}\right)$ and $\varepsilon^{0}>0$, there exist $N^{0}$ and $\delta^{0}>0$ such that

$$
\left|\int_{M} \mu_{n N}\left(d \sigma \mid \beta, v_{j, \varepsilon, \beta}^{*}, \sigma_{N}^{\prime}\right)-\int_{M} \mu_{n \infty}\left(d \sigma \mid \beta, v_{j, \varepsilon, \beta}^{*}\right)\right|<\varepsilon^{0}
$$

if $N>N^{0}$ and $\left|h\left(\sigma_{N}^{\prime}\right)\right|<\delta^{0}$. Therefore it is sufficient to prove that $h\left(\sigma_{N}^{\prime}\right)_{N \rightarrow \infty} 0$ in probability. Let us fix a number $N_{0}$, for instance, $N_{0}=1$. Then by the DLR condition the quantity $h\left(\sigma_{N_{0}}^{\prime}\right)=\sum_{j \notin V_{N_{0}}, 0} d^{-a}(i, j) \sigma^{\prime}(j)$ is finite with probability 1 . But $h\left(\sigma_{N}^{\prime}\right)=\sum_{j \notin V_{N_{0}, 0}} d^{-a}(i, j) \sigma^{\prime}(j)$ is the tail of the sum $\sum_{j \notin V_{N_{0}, 0}} d^{-a}(i, j) \sigma^{\prime}(j)$ so it tends to zero in probability when $N \rightarrow \infty$. The theorem is proved.

Proof of Theorem 5. As for Theorem 2, we have to prove the convergence of the functions $f_{n N}(x) / f_{n N}(0)$ to a limit when $N \rightarrow \infty$, where

$$
f_{n N}(x)=T_{n+1} \ldots T_{N} 1=\exp \left(\alpha x^{2}\right) S_{n+1} \ldots S_{N} \exp \left(-\alpha x^{2}\right) .
$$

The kernel of the operator $S_{k}$ is

$$
\begin{gathered}
S_{k}(x, y)=\exp \left[-A(x-y)^{2}-B x^{2}-B y^{2}-\varphi^{(k)}\left(\frac{2}{\sqrt{c}} y-x\right)\right] \\
p^{(k)}(x)=\exp \left[-a_{0} x^{2}-\varphi^{(k)}(x)\right] .
\end{gathered}
$$

By the conditions (2.7), (2.8), $S_{k \rightarrow \infty} S$ and

$$
\left\|S_{k}-S\right\|<C \lambda^{k}
$$

$0<\lambda<1$. Let $\lambda_{0}$ be the largest eigenvalue of the operator $S$ and

$$
\bar{S}_{k}=\lambda_{0}^{-1} S_{k}, \bar{S}=\lambda_{0}^{-1} S .
$$


We shall prove now the convergence in $L^{2}$ (and hence in $\mathscr{S}$ ) of the functions $g_{n N}(x)=\bar{S}_{n+1} \ldots \bar{S}_{N} \exp \left(-\alpha x^{2}\right)$ when $N \rightarrow \infty$.

\section{Lemma 3.2.}

(i) $\sup _{N, n}\left\|\bar{S}_{N+1} \ldots \bar{S}_{N+n}\right\|=F<\infty$.

(ii) There exists $N_{0}$ such that for $N \geqq N_{0}$,

$$
\sup _{n}\left\|\bar{S}_{N+1} \ldots \bar{S}_{N+n}-\bar{S}^{n}\right\| \leqq \lambda^{N / 2}
$$

Proof. By (3.17), (3.17') we have that

$$
\sup _{n}\left\|\bar{S}^{n}\right\|=F^{0}<\infty
$$

thus (i) follows from (ii), so it is sufficient to prove (ii). Define $F_{0}^{(N)}=0$,

$$
F_{n}^{(N)}=\sup _{M \geqq N}\left\|\bar{S}_{M+1} \ldots \bar{S}_{M+n}-\bar{S}^{n}\right\|,
$$

$n=1,2, \ldots$ As

$$
\bar{S}_{M+1} \ldots \bar{S}_{M+n}=\sum_{j=1}^{n} \bar{S}^{j-1}\left(\bar{S}_{M+j}-\bar{S}\right) \bar{S}_{M+j+1} \ldots \bar{S}_{M+n}
$$

then

$$
\begin{aligned}
\left\|\bar{S}_{M+1} \ldots \bar{S}_{M+n}-\bar{S}^{n}\right\| & \leqq \sum_{j=1}^{n}\left\|\bar{S}^{j-1}\right\| \cdot\left\|\bar{S}_{M+j}-\bar{S}\right\| \cdot\left\|\bar{S}_{M+j+1} \ldots \bar{S}_{M+n}\right\| \\
& \leqq F^{0} \sum_{j=1}^{n} C \lambda^{M+j}\left(F_{n-j}^{(N)}+F^{0}\right) \\
& \leqq C_{1} \lambda^{N}+C_{2} \lambda^{N} \sum_{j=1}^{n} \lambda^{j} F_{n-j}^{(N)},
\end{aligned}
$$

if $M \geqq N$, where $C_{1}=\left(F^{0}\right)^{2} C, C_{2}=F^{0} C$. Therefore

$$
F_{n}^{(N)} \leqq C_{1} \lambda^{N}+C_{2} \lambda^{N} \sum_{j=1}^{n} \lambda^{j} F_{n-j}^{(N)} .
$$

We assert that for large $N$,

$$
F_{n}^{(N)}<\lambda^{N / 2}
$$

This is trivial for $F_{0}^{(N)}=0$. Assume it is true for $F_{0}^{(N)}, \ldots, F_{n-1}^{(N)}$. Then by (3.52),

$$
F_{n}^{(N)} \leqq C_{1} \lambda^{N}+C_{2} \lambda^{N} \sum_{j=1}^{n} \lambda^{j} \lambda^{N / 2}<C_{3} \lambda^{N}<\lambda^{N / 2},
$$

if $N$ is large. Thus (3.53) is proved. Now,

$$
\begin{aligned}
\sup _{n}\left\|\bar{S}_{N+1} \ldots \bar{S}_{N+n}-\bar{S}^{n}\right\| & \leqq \sup _{M \geqq N} \sup _{n}\left\|\bar{S}_{M+1} \ldots \bar{S}_{M+n}-\bar{S}^{n}\right\| \\
& =\sup _{n} F_{n}^{(N)}<\lambda^{N / 2},
\end{aligned}
$$

which proves the lemma. 
Let us return to the proof of the theorem. We assert the following:

(i) $g_{n N}(x)_{N \rightarrow \infty} g_{n}(x)$ in $\mathscr{S}\left(\mathbb{R}^{1}\right)$,

(ii) $\lim _{n \rightarrow \infty} g_{n}(x)=\mu e_{0}(x), \quad \mu>0$.

Assuming these statements we finish the proof of the theorem. They ensure the existence of a limit Gibbs distribution. As in the proof of Theorem 2 its finite dimensional distributions have the form const $\cdot \exp \left(\alpha \xi_{n}^{2}\right) g_{n}\left(\xi_{n}\right) \mu\left(d \sigma \mid v_{n}, 0\right)$. Under the renormalization transformation they are changed to const $\exp \left(\alpha \xi_{n}^{2}\right) g_{n+1}\left(\xi_{n}\right)$ $\mu\left(d \sigma \mid v_{n+1}, 0\right)$. As $\lim _{n \rightarrow \infty} g_{n}(x)=\mu e_{0}(x)$ and $\lim _{n \rightarrow \infty} v_{n}=v^{*}$, the iterations of the renormalization transformations have a limit const $\cdot \exp \left(a \xi_{n}^{2}\right) \cdot e_{0}(x) \mu\left(d \sigma \mid v^{*}, 0\right)$ which coincides with the finite dimensional distributions of the self-similar random field $\mu\left(d \sigma \mid v^{*}\right)$. Thus the theorem is proved and it remains to establish (i), (ii).

Let $n<M<N$. We have:

$$
\begin{aligned}
g_{n N}= & \bar{S}_{n+1} \ldots \bar{S}_{N} g=g_{n N}^{(1)}+g_{n N}^{(2)}, \\
g_{n N}^{(1)}= & \bar{S}_{n+1} \ldots \bar{S}_{M} \bar{S}^{N-M} g ; g_{n N}^{(2)}=\bar{S}_{n+1} \ldots \bar{S}_{M}\left(\bar{S}_{M+1} \ldots \bar{S}_{N}\right. \\
& \left.-\bar{S}^{N-M}\right) g ; g=\exp \left(-\alpha x^{2}\right)=\mu e_{0}+g^{\prime} .
\end{aligned}
$$

By (3.18), $\left\|\bar{S}^{N-M} g-\mu e_{0}\right\| \leqq C \varrho^{N-M}, 0<\varrho<1$, so

$$
\begin{aligned}
\left\|g_{n N}^{(1)}-g_{n N^{\prime}}^{(1)}\right\| & \leqq\left\|\bar{S}_{n+1} \ldots \bar{S}_{M}\right\| \cdot\left\|\bar{S}^{N-M} g-\bar{S}^{N^{\prime}-M} g\right\| \\
& \leqq C_{1}\left(\varrho^{N-M}+\varrho^{N^{\prime}-M}\right),
\end{aligned}
$$

if $N, N^{\prime} \geqq M$. Moreover by Lemma 3.2 above $g_{n N}^{(2)}, g_{n N^{\prime}}^{(2)} \rightarrow 0$ when $M \rightarrow \infty$. So $g_{n N}-g_{n N^{\prime}} \rightarrow 0$ when $N, N^{\prime} \rightarrow \infty$ as we can take $M=\left[(1 / 2) \min \left\{N, N^{\prime}\right\}\right]$ and then $g_{n N}^{(1)}-g_{n N^{\prime}}^{(1)} \rightarrow 0$ and $g_{n N}^{(2)}-g_{n N^{\prime}}^{(2)} \rightarrow 0$. Thus the sequence $g_{n N}$ satisfies the Cauchy condition when $N \rightarrow \infty$ and so it converges to a limit. Now by the lemma,

$$
g_{n N}-\bar{S}^{N-n} g=\left(\bar{S}_{n+1} \ldots \bar{S}_{N}-\bar{S}^{N-n}\right) g \rightarrow 0
$$

when $n \rightarrow \infty, N \geqq n$, and moreover $\bar{S}^{N-n} g \rightarrow \mu e_{0}$ when $N-n \rightarrow \infty$. Therefore $g_{n}=\lim _{N \rightarrow \infty} g_{n N_{n} \rightarrow \infty} \mu e_{0}$, which was stated in (ii). This completes the proof.

Acknowledgements. The author thanks R.L.Dobrushin very much for useful discussions on the uniqueness theorem of the limit Gibbs distributions, Ya.G. Sinai for attentive reading of the manuscript and valuable remarks and N. Angelescu for his help in preparing this text for publication.

\section{References}

1. Bleher, P.M., Sinai, Ya.G.: Investigation of the critical point in models of the type of Dyson's hierarchical models. Commun. Math. Phys. 33, 23-42 (1973)

2. Bleher, P.M., Sinai, Ya.G.: Critical indices for Dyson's asymptotically hierarchical models. Commun. Math. Phys. 45, 247-278 (1975)

3. Collet, P., Eckmann, J.-P.: A renormalization group analysis of the hierarchical model in statistical mechanics. In : Lecture Notes in Physics, Vol. 74, pp. 1-199. Berlin, Heidelberg, New York : Springer 1978

4. Sinai, Ya.G.: Self-similar probability distributions. Teor. Veroyatn. Ee Primen. 21, 63-80 (1976) 
5. Dobrushin, R.L.: Description of random field with help of conditional probabilities and conditions of its regularity. Teor. Veroyatn. Ee Primen. 2, 201-229 (1968)

6. Lanford, O.E., Ruelle, D.: Observables at infinity and states with shortrange correlations in statistical mechanics. Commun. Math. Phys. 3, 194-215 (1968)

7. Kim, D., Thompson, C.J. : Critical properties of Dyson's hierarchical model. II, III. J. Phys. A11, 375-384, 385-401 (1978)

8. Bleher, P.M.: A second order phase transition in some ferromagnetic models. Proc. Mosc. Math. Society 33, 155-222 (1975)

9. Kato, T.: Perturbation theory for linear operators. Berlin, Heidelberg, New York: Springer 1966

Communicated by Ya.G.Sinai

Received December 10, 1981 